\title{
Cáncer en pacientes con enfermedad tromboembólica venosa
}

\author{
J. M. CALVO ROMERO, E. M. LIMA RODRÍGUEZ ${ }^{1}$ \\ Servicio de Medicina Interna. Hospital Ciudad de Coria. ${ }^{~}$ Área de Salud. Coria. Cáceres
}

CANCER IN PATIENTS WITH VENOUS THROMBOEMBOLIC DISEASE

\begin{abstract}
RESUMEN
Objetivo: Estudiar las características de los pacientes con enfermedad tromboembólica venosa (ETV) y cáncer en nuestro medio.

Métodos: Estudio observacional prospectivo de todos los pacientes diagnosticados mediante métodos objetivos de ETV en el único hospital de un área rural entre noviembre de 2002 y abril de 2005.

Resultados: Se diagnosticaron 94 casos de ETV. La edad media fue 71,9 años (rango 35-94 años), y 52 (55,3\%) fueron mujeres. El tiempo medio de seguimiento con tratamiento anticoagulante fue 7,1 meses (rango 0-29 meses). Diecisiete casos $(18,1 \%)$ tuvieron cáncer. Once casos $(64,7 \%)$ tuvieron un adenocarcinoma, y $7(41,2 \%)$ presentaban metástasis a distancia. El diagnóstico de cáncer se estableció después del diagnóstico de la ETV en 2 (2,5\%) de 79 casos sin cáncer previo. La frecuencia de eventos adversos (fallecimiento, hemorragia grave y recidiva tromboembólica) fue significativamente mayor en los pacientes con cáncer $(64,7$ vs. $9,1 \%, \mathrm{p}<0,001)$.

Conclusiones: Casi una quinta parte de los pacientes con ETV en nuestro medio tienen cáncer, más frecuentemente un adenocarcinoma. El cáncer se diagnostica infrecuentemente después de la ETV. El cáncer en la ETV se asocia a un pobre pronóstico, con una considerable frecuencia de eventos adversos.
\end{abstract}

PALABRAS CLAVE: Cáncer. Enfermedad tromboembólica venosa. Tromboembolia venosa.

\begin{abstract}
Objective: To study the characteristics of patients with venous thromboembolic disease (VTD) and cancer in our area.

Methods: Prospective observational study of all patients diagnosed by objective methods of VTD in the only hospital in a rural area from November 2002 to April 2005.

Results: Ninety-four patients were diagnosed of VTD. The median age was 71.9 years (range 35-94 years), and 52 (55.3\%) were women. The median follow-up with anticoagulant therapy was 7.1 months (range 0-29 months). Seventeen patients (18.1\%) had cancer. Eleven cases (64.7\%) had an adenocarcinoma, and $7(41.2 \%)$ had distant metastases. Two $(2.5 \%)$ of 79 cases without prior cancer were diagnosed of cancer after the diagnosis of VTD. The frequency of adverse events (death, major hemorrhage and thromboembolic recurrence) was higher in patients with cancer $(64.7 v s .9 .1 \%, p<0.001)$.

Conclusions: Almost one fifth of patients with VTD in our area had cancer, more frequently an adenocarcinoma. Cancer is infrequently diagnosed after the diagnosis of VTD. Cancer in VTD is associated to a poor prognosis, with a remarkable frequency of adverse events.
\end{abstract}

KEY WORDS: Cancer. Venous thromboembolic disease. Venous thromboembolism.

Calvo Romero JM, Lima Rodríguez EM. Cáncer en pacientes con enfermedad tromboembólica venosa. An Med Interna (Madrid) 2007; 24: $571-573$.

\section{INTRODUCCIÓN}

El riesgo de enfermedad tromboembólica venosa (ETV), que incluye la trombosis venosa profunda (TVP) y el tromboembolismo pulmonar (TEP), está aumentado en los pacientes con cáncer $(1,2)$. En este artículo se describen las características de los pacientes con cáncer y ETV de una serie de casos consecutivos diagnosticados en un área rural.

\section{MATERIAL Y MÉTODOS}

Se realizó un estudio observacional prospectivo de todos los pacientes diagnosticados de ETV (TEP y/o TVP) mediante métodos objetivos entre noviembre de 2002 y abril de 2005 en el único hospital de un área rural de Extremadura. El diagnóstico de TVP se estableció mediante ecografía-doppler venosa o tomografía computarizada (TC). El diagnóstico de TEP se esta-

Trabajo aceptado: 28 de agosto de 2007 
bleció mediante gammagrafía pulmonar de ventilación/perfusión de alta probabilidad, TC o la asociación de un cuadro clínico compatible con TEP y la demostración de trombosis venosa profunda mediante ecografía-doppler venosa o TC. En todos los pacientes se programó un seguimiento de al menos 3 meses con tratamiento anticoagulante.

Se definió comorbilidad como la existencia de patología crónica grave pulmonar o cardíaca, demencia avanzada u otras enfermedades graves, excluido el cáncer. Se definió evento adverso como el fallecimiento, la hemorragia grave o la recidiva tromboembólica. Se consideró hemorragia grave si causó un descenso de la hemoglobina superior a $2 \mathrm{~g} / \mathrm{dl}$, precisó de la transfusión de 2 o más concentrados de hematíes, o la localización fue retroperitoneal o en sistema nervioso central.

El análisis estadístico se realizó utilizando la prueba chicuadrado y el test exacto de Fisher, cuando alguno de los valores esperados fue menor de 5, para la comparación de proporciones y la prueba de la t de Student para la comparación de medias. Se consideró estadísticamente significativo un valor de $\mathrm{p}$ inferior a 0,05 .

\section{RESULTADOS}

En el periodo estudiado se diagnosticaron de ETV un total de 94 pacientes: 40 con TEP y 54 con TVP. La edad media fue 71,9 años (rango 35-94 años), y 52 (55,3\%) fueron mujeres. El tiempo medio de seguimiento con tratamiento anticoagulante fue 7,1 meses (rango 0-29 meses). Diecisiete pacientes $(18,1 \%)$ tuvieron cáncer. Diez $(58,8 \%)$ de los 17 pacientes con cáncer tuvieron una TVP aislada. Todas las TVP se localizaron en miembros inferiores. Ninguno de los 17 pacientes tuvo el antecedente de cirugía mayor en los 2 últimos meses. En 15 casos el diagnóstico de cáncer precedió al diagnóstico de la ETV. El diagnóstico de cáncer se estableció después del diagnóstico de la ETV en $2(2,5 \%)$ de un total de 79 casos sin cáncer previo. Los tipos histológicos fueron: adenocarcinoma (11 casos), carcinoma epidermoide (2 casos), carcinoma microcítico (1 caso) y no determinado (3 casos). Las localizaciones se describen en la tabla I. Siete casos $(41,2 \%)$ presentaban metástasis a distancia, y $3(17,6 \%)$ estaban en tratamiento con quimioterapia.

Todos los pacientes recibieron en la fase aguda heparina de bajo peso molecular a dosis terapéuticas. Ningún paciente fue tratado con trombolíticos o un filtro en la vena cava infe-

TABLA I

LOCALIZACIÓN DE LOS CÁNCERES DE LA SERIE

\begin{tabular}{lc}
\hline & $n=17$ \\
\hline Pulmón & 3 \\
Primario desconocido & 3 \\
Próstata & 2 \\
Mama & 2 \\
Ovario & 1 \\
Endometrio & 1 \\
Estómago & 1 \\
Colon & 1 \\
Vejiga & 1 \\
Orofaringe & 1 \\
Abdominal & 1 \\
\hline
\end{tabular}

rior. Como tratamiento a largo plazo, todos los pacientes recibieron acenocumarol, excepto 9 pacientes que fueron tratados con heparina de bajo peso molecular (3 de ellos con cáncer). Diez pacientes con cáncer fallecieron durante el seguimiento: 5 por progresión del cáncer, 3 por recidiva tromboembólica, 1 por hemorragia y 1 por una enfermedad pulmonar obstructiva crónica agudizada. No se realizó autopsia en ningún caso. Cuatro pacientes $(23,5 \%)$ tuvieron una hemorragia grave durante el seguimiento: 3 de localización digestiva y 1 orofaríngea. En la tabla II se comparan las características de los pacientes con y sin cáncer.

TABLA II

COMPARACIÓN DE LAS CARACTERÍSTICAS DE LOS PACIENTES CON Y SIN CÁNCER

\begin{tabular}{lccc}
\hline & $\begin{array}{c}\text { Cáncer } \\
n=17\end{array}$ & $\begin{array}{c}\text { No cáncer } \\
n=77\end{array}$ & $p$ \\
\hline Edad (años) & $72,4 \pm 8,7$ & $71,9 \pm 12,3$ & NS \\
Edad > 70 años & $11(64,7 \%)$ & $52(67,5 \%)$ & NS \\
Mujer & $8(47,1 \%)$ & $44(57,1 \%)$ & NS \\
Tromboembolismo pulmonar & $7(41,2 \%)$ & $33(42,9 \%)$ & NS \\
Comorbilidad & $7(41,2 \%)$ & $24(31,2 \%)$ & NS \\
Enfermedad cardiopulmonar & $6(35,3 \%)$ & $16(20,8 \%)$ & NS \\
Inmovilización & $4(23,5 \%)$ & $29(37,7 \%)$ & NS \\
ETV previa & 0 & $5(6,5 \%)$ & NS \\
Insuficiencia renal crónica* & $4(23,5 \%)$ & $8(10,4 \%)$ & NS \\
Tiempo hasta el diagnóstico (días) & $7,6 \pm 8,9$ & $8,5 \pm 16,3$ & NS \\
Hemorragia grave & $4(23,5 \%)$ & $2(2,6 \%)$ & 0,009 \\
Recidiva & $3(17,6 \%)$ & $1(1,3 \%)$ & 0,01 \\
Fallecimiento & $10(58,8 \%)$ & $6(7,8 \%)$ & $<0,001$ \\
Eventos adversos & $11(64,7 \%)$ & $7(9,1 \%)$ & $<0,001$ \\
\hline
\end{tabular}

ETV: enfermedad tromboembólica venosa; ${ }^{*}$ : creatinina plasmática $>1,5$ $\mathrm{mg} / \mathrm{dl} ; \mathrm{NS}$ : no significación estadística $(\mathrm{p} \geq 0,05)$.

\section{DISCUSIÓN}

El cáncer y la quimioterapia pueden causar hipercoagulabilidad y lesión del endotelio vascular, de manera que el riesgo de ETV está aumentado $(1,2)$. Casi una quinta parte de los pacientes con ETV de nuestro estudio tuvieron cáncer. En sólo poco más del $2 \%$ de nuestros pacientes con ETV sin cáncer previo se identificó un cáncer después del diagnóstico de la ETV con un seguimiento medio de más de 7 meses con tratamiento anticoagulante. En un análisis retrospectivo de una serie española, un $21,8 \%$ de 674 pacientes con ETV tenían previamente un cáncer y un 3,4\% fueron diagnosticados de cáncer en el momento del diagnóstico de la ETV o tras dicho diagnóstico (3). En el registro RIETE, de un total de 14.391 pacientes con ETV un $20 \%$ tuvieron cáncer (4). En base a los datos de un estudio poblacional, se ha estimado que el $18 \%$ de los casos de ETV son atribuibles al cáncer (5). La ETV puede ser la primera manifestación de un cáncer oculto. No obstante, parece que el cáncer se diagnostica infrecuentemente en los primeros meses tras la ETV. Además, transcurridos 6 meses del diagnóstico de la ETV, el riesgo de cáncer se normaliza (6).

La incidencia de ETV es mayor en los pacientes con cáncer metastático y en los que reciben quimioterapia $(7,8)$. Aproximadamente un $40 \%$ de los pacientes de una gran serie con el diagnóstico de cáncer simultáneo o en el año posterior a la ETV tuvieron metástasis a distancia (9). Casi la mitad de nuestros pacientes con ETV y cáncer presentaban metástasis a 
distancia. Igualmente casi una quinta parte estaban en tratamiento con quimioterapia. Se ha sugerido que la ocurrencia de ETV podría ser un marcador de mayor actividad del cáncer (2). El tipo histológico más frecuente en nuestro estudio fue el adenocarcinoma y las localizaciones fueron diversas, hallazgos coincidentes con lo descrito en la literatura $(1,2)$.

El cáncer diagnosticado en el momento de una ETV o en el año siguiente se asocia a una supervivencia reducida (8). Además, la recidiva tromboembólica y la hemorragia grave son considerablemente más frecuentes en los pacientes con ETV y cáncer que en los que no tienen cáncer (2,9-11). La frecuencia de eventos adversos (fallecimiento, hemorragia grave y recidiva tromboembólica) fue considerable en nuestros pacientes con cáncer, superior al $60 \%$ tras una media de más de 7 meses de seguimiento con tratamiento anticoagulante, y significativamente mayor que en los pacientes sin cáncer. En un estudio norteamericano, el cáncer activo fue identificado

\section{Bibliografía}

1. Lee AY, Levine MN. Venous thromboembolism and cancer: risks and outcomes. Ciculation 2003; 107 (Supl. 1): 17-21.

2. Lecumberri R, Feliu J, Rocha E. Tromboembolia venosa en pacientes con cáncer. Med Clin (Barc) 2006; 127: 22-32.

3. Monreal M, Fernández-Llamazares J, Perandreu J, Urrutia A, Sahuquillo JC, Contel E. Occult cancer in patients with venous thromboembolism: Which patients, which cancers. Thromb Haemost 1997; 78: 1316-8.

4. Monreal M, Falga C, Valdes M, Suárez C, Gabriel F, Tolosa C, et al. Fatal pulmonary embolism and fatal bleeding in cancer patients with venous thromboembolism: findings from the RIETE registry. J Thromb Haemost 2006; 4: 1950-6.

5. Heit JA, O'Fallon WM, Petterson TM, Lohse CM, Silverstein MD, Mohr DN, et al. Relative impact of risk factors for deep vein thrombosis and pulmonary embolism. Arch Intern Med 2002; 162: 1245-8.

6. Sørensen HT, Mellemkjær L, Steffensen FH, Olsen JH, Nielsen GL. The risk of a diagnosis of cancer after primary deep vein thrombosis and pulmonary embolism. N Engl J Med 1998; 338: 1169-73.

7. Heit JA, Silverstein MD, Mohr DN, Petterson TM, O'Fallon WM, Mel- como el único factor pronóstico de mortalidad y de recurrencia en pacientes mayores de 45 años con ETV (9). En un estudio italiano de 842 pacientes con ETV, la mayoría con TVP, un $20 \%$ de los pacientes con cáncer presentaron recidiva tromboembólica y un $12 \%$ hemorragia grave en los primeros 12 meses, cifras que podemos considerar similares a las de nuestro estudio, y que fueron significativamente mayores que en los pacientes sin cáncer (10). En el registro RIETE, el TEP y la hemorragia fatales fueron más frecuentes en los pacientes con ETV y cáncer que en los pacientes sin cáncer (4).

Nuestros resultados sugieren que la elevada mortalidad en los pacientes con cáncer y ETV es debida a complicaciones relacionadas con la ETV (recurrencia y hemorragia) y sobre todo a la progresión del cáncer. No obstante, limitaciones de nuestro estudio son que no se realizaron autopsias, y que por tanto no tenemos certeza de las causas de los fallecimientos, y que el número de casos de la serie es reducido.

ton LJ III. Risk factors for deep vein thrombosis and pulmonary embolism: A population-based case-control study. Arch Intern Med 2000; 160: 809-15.

8. Sørensen HT, Mellemkjær L, Olsen JH, Baron JA. Prognosis of cancers associated with venous thromboembolism. N Engl J Med 2000; 343: 1846-50.

9. Cushman M, Tsai AW, White RH, Heckbert SR, Rosamond WD Enright P, et al. Deep vein thrombosis and pulmonary embolism in two cohorts: the longitudinal investigation of thromboembolism etiology. Am J Med 2004; 117: 19-25.

10. Prandoni P, Lensing AW, Piccioli A, Bernardi E, Simioni P, Girolami $\mathrm{B}$, et al. Recurrent venous thromboembolism and bleeding complications during anticoagulant treatment in patients with cancer and venous thrombosis. Blood 2002; 100: 3484-8.

11. Hutten BA, Prins MH, Gent M, Ginsberg J, Tijssen JG, Buller HR. Incidence of recurrent thromboembolic and bleeding complications among patients with venous thromboemblism in relation to both malignancy and achieved international normalized ratio: a retrospective analysis. $\mathbf{J}$ Clin Oncol 2000; 18: 3078-83. 